\title{
REVIEW
}

\section{Intestinal immune compartmentalization: implications of tissue specific determinants in health and disease}

\author{
Hailey Brown ${ }^{1}$ and Daria Esterházy $\mathbb{D}^{1,2}$
}

The emerging concept of tissue specific immunity has opened the gates to new inquiries into what factors drive immune cell niche adaptation and the implications on immune homeostasis, organ specific immune diseases, and therapeutic efficacy. These issues are particularly complicated at barrier sites, which are directly exposed to an ever-changing environment. In particular, the gastrointestinal $(\mathrm{Gl})$ tract faces even further challenges given the profound functional and structural differences along its length, raising the possibility that it may even have to be treated as multiple organs when seeking to answer these questions. In this review, we evaluate what is known about the tissue intrinsic and extrinsic factors shaping immune compartments in the intestine. We then discuss the physiological and pathological consequences of a regionally distinct immune system in a single organ, but also discuss where our insight into the role of the compartment for disease development is still very limited. Finally, we discuss the technological and therapeutic implications this compartmentalization has. While the gut is perhaps one of the most intensely studied systems, many of these aspects apply to understanding tissue specific immunity of other organs, most notably other barrier sites such as skin, lung, and the urogenital tract.

Mucosal Immunology (2021) 14:1259-1270; https://doi.org/10.1038/s41385-021-00420-8

\section{THE INTESTINAL IMMUNE NICHES-ALMOST MULTIPLE ORGANS}

The intestine is a continuous tubular structure that spans from the stomach to the anus and is responsible for most nutrient and water absorption within an organism. Though a single continuous unit, the intestine can be broken down anatomically into the small and large intestine and even further subdivided within each region based on physical and functional properties. The small intestine (SI) includes the duodenum, jejunum, and ileum whereas the large intestine (LI) includes the cecum, colon, rectum, and anus. The intestinal immune system must not only contend with the continuous exposure to food, commensal microbiota and pathogens, but respond appropriately according to tissue differences across the intestine. Importantly, the entire intestine, inclusive of its lymph nodes, is considered a by default immunosuppressive organ overall compared to most other tissues, indicating that a state of tolerance to food and commensals yet vigilance toward pathogens was an evolutionarily stable strategy. Historically the gut immune system has been examined as small versus large intestine, based on obvious differences in digestive function. However, in recent years it has become apparent that the immunological niches of the Gl tract differ between more refined functional compartments, necessitating careful study of each in their own right to understand the consequences on intestinal immune homeostasis.

The regional properties of the intestine with respect to immune populations at each site have been extensively reviewed elsewhere (see refs. ${ }^{1,2}$ ), and we strongly suggest consulting these reviews complementarily to ours. Relative immune cell frequencies as well as gene expression profiles differ, in some cases majorly, between the small and large intestine, summarized in Table 1. However, a systematic comparison of all $\mathrm{CD}_{4} 5^{+}$immune cells across each intestinal segment in both mice and humans is lacking. Hallmarks of the SI immune system include: A decreasing gradient proximally to distally of luminal $\lg \mathrm{A}$, intraepithelial lymphocytes (IELs), polyclonal Th17 CD4 and CD8 T cells, natural killer cells, type 2 innate lymphocyte cells (ILCs), eosinophils, mast

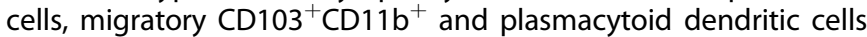
(DCs) in the lamina propria, and an increasing gradient of $\mathrm{RORgt}^{+}$ peripheral regulatory $\mathrm{T}$ ( $\mathrm{pTreg}$ ) cells and ILC3s. In contrast, the $\mathrm{LI}$ exhibits higher frequencies of IgA plasma cells, RORgt ${ }^{+}$Treg and overall Treg cells, much lower frequencies of IELs and granulocytes than the small intestine, and is rather enriched for macrophages and $C D 103^{+} C D 11 b^{-}$DCs (Table 1). Frequencies of B cells, T cells and DCs in the draining lymph nodes (LNs) are not strikingly different within a mouse, but rather the gene expression profiles reveal the regionalization. Unsurprisingly, the microbiota influences a number of immune cell frequencies within the gut, notable examples include the severely reduced levels of induced IELs, ${ }^{3}$ Th17 cells ${ }^{4}$ and $\operatorname{lgA}^{5}$ in germ free mice, and the ratio of

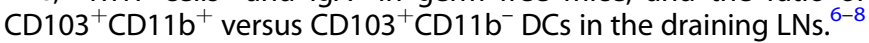

In this review, we will highlight key features of each organ that may help shape each of these immune niches, discuss the more subtle properties of the immune cells in each compartment that point to an adaptation to the milieu, and highlight the potential ramifications of these tissue-immune milieus in relation to intestinal diseases.

Intestinal compartmentalization implies (1) a regional containment and (2) location specific differences. It enables the immune system to adapt to the specific needs of each site set by the tissue function and site-specific extrinsic challenges. Immune function including priming, effector cell differentiation and tissue maintenance can therefore be tailored to each niche. By operating in compartments, the immune

${ }^{1}$ Committee on Immunology, University of Chicago, Chicago, IL, USA and ${ }^{2}$ Department of Pathology, University of Chicago, Chicago, IL, USA

Correspondence: Daria Esterházy (desterhazy@bsd.uchicago.edu)

Received: 10 December 2020 Revised: 5 May 2021 Accepted: 24 May 2021

Published online: 1 July 2021 
Table 1. Immune cell profiles along the intestine.

\begin{tabular}{|c|c|c|}
\hline & Small intestine & Large intestine \\
\hline B cells & + & +++ \\
\hline $\lg \mathrm{A}^{+}$plasma cells & +++ & +++ \\
\hline T cells & + & + \\
\hline Th17 & +++ & + \\
\hline Foxp3 ${ }^{+}$Treg & + & +++ \\
\hline Th1 & + & + \\
\hline APCs & + & ++ \\
\hline 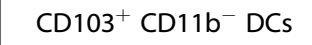 & + & ++ \\
\hline $\mathrm{CD}_{103^{+}} \mathrm{CD} 11 \mathrm{~b}^{+} \mathrm{DCs}$ & ++ & + \\
\hline $\mathrm{CD}_{11 \mathrm{~b}^{+}}$Macrophages & +++ & +++ \\
\hline IELs & +++ & + \\
\hline $\mathrm{TCR} \alpha \beta^{+} \mathrm{CD} 4^{+}$ & + & ++ \\
\hline $\mathrm{TCR} \alpha \beta^{+} \mathrm{CD} 8 \alpha \alpha^{+}$ & +++ & + \\
\hline $\mathrm{TCR} \alpha \beta^{+} \mathrm{CD} 8 \alpha \beta^{+}$ & ++ & + \\
\hline $\mathrm{TCR} \gamma \delta^{+}$ & ++ & + \\
\hline Eosinophils & +++ & + \\
\hline Mast cell & +++ & + \\
\hline ILCS & + & \\
\hline ILC1 & + & + \\
\hline ILC2 & ++ & + \\
\hline ILC3 & +++ & + \\
\hline
\end{tabular}

system may generate multiple immune outcomes, even with opposite goals e.g., tolerance or inflammation, simultaneously. The generation of unique immunologic niches within the intestine is influenced by a combination of tissue intrinsic properties, extrinsic environmental factors, and regionalized immune populations. We will first describe the factors that may influence the generation of a regional immune system and illustrate- in a by no means exhaustive way- how this regionality may explain niche-selective diseases of the $\mathrm{Gl}$ tract. We will then discuss more broadly what the implications of a regionalized intestinal immune system are for physiological versus pathological conditions, but also for therapeutic and technological hurdles and opportunities. Finally, we will point out where the limits of our knowledge stand.

For the purposes of this review, we will omit the oral cavity, stomach and omentum, and consider the Gl tract with respect to five major immune compartments: (1) the proximal SI, consisting of the duodenum and jejunum, (2) the distal SI (the ileum), (3) the $\mathrm{LI}$ (the colon), and the structures that further support intestinal immune compartmentalization, (4) the mesentery, and (5) the gutdraining lymph nodes ( $\mathrm{gLNs}$ ) and gut associated lymphoid tissue (GALT; Fig. 1).

\section{THE PROXIMAL SMALL INTESTINE (SI)}

\section{Interplay between intrinsic and extrinsic factors}

Positioned adjacent to the stomach, the duodenum and jejunum are adapted for efficient absorption of the high concentration of dietary derived nutrients. Long villi within the proximal intestine increase the surface area for absorption, with villus length decreasing from the duodenum to the ileum, mirroring the absorptive capacity of the small intestine. While seminal work on intraepithelial cells (IELs) performed in the jejunum of Celiac and gastric bypass patients has contributed to our understanding of these cells, other jejunum-specific immunological features are currently understudied, and as such this gut segment is hitherto primarily described as an intermediary between duodenum and ileum. Together the duodenum and jejunum account for about $75 \%$ of the small intestine in mice, and slightly less in humans where the duodenum is shorter in proportion. With only a thin mucus layer separating the large surface area of the epithelium from luminal bacteria, the proximal SI immune system must maintain the barrier without hindering absorption. Aided by higher oxygen levels, acidic $\mathrm{pH}$, a faster transit time as well as high amounts of antimicrobial peptides, the proximal $\mathrm{SI}$ is able to limit the types and amounts of bacteria present, while still ensuring nutrient uptake. In addition, the duodenum has the highest concentrations of luminal $\lg \mathrm{A}$, decreasing along the length of the gut, ${ }^{9}$ possibly in part due to enterohepatic recirculation of $\lg \mathrm{A}$ leading to high biliary levels. ${ }^{10}$

The adaptations of the proximal intestine that facilitate nutrient absorption have the inverse effect on commensal microbe survival, resulting in a severely reduced microbial load, $10^{1}-10^{3}$ bacteria per $\mathrm{ml}$ content, compared to the ileum and $\mathrm{LI}$ where it can reach $10^{10}$ per $\mathrm{ml}$. Owing to increased protection from the harsh elements via their thick cell wall, gram-positive bacteria such as Lactobacillus are enriched in the duodenal microbiome. ${ }^{11}$ Alterations to the duodenal microbiome have been associated with Gl symptoms, ${ }^{12}$ but given the relatively sparse microbial community and difficulty of obtaining samples less attention has been paid to this region in general.

The regionalized input of pancreas- and gallbladder-derived enzymes and primary bile acids not only have important functions in digestion, aiding in macromolecule break down and lipid emulsification, respectively, but help shape the immune landscape of the proximal SI. In addition to being antimicrobial, bile acids can undergo secondary modifications by the resident microbiota, acting as signaling molecules to affect $T$ helper differentiation and infection tropism. ${ }^{13-15}$ While bile acids are more frequently studied in the distal gut where they are more likely to be bioconverted, their receptors are also expressed in the proximal intestine and thus may still have important roles here.

The local concentration of bile acids facilitates the highest lipid absorption by enterocytes in both the duodenum and the jejunum. Long chain free fatty acids, triglycerides and cholesterol are packaged into chylomicrons and released for uptake by lymphatic vessels, being too large for blood capillaries. The lymphatic vessels, known as lacteals, within the villi are not only longer in the duodenum due to the difference in villi length, but also more frequent, with an average of two lacteals per villus compared to one per villus in the ileum. ${ }^{16}$ Whether the high levels of lipids in the proximal SI influence immune responses, either through consequent effects on lymph flow or lipid concentrations within lymph nodes, is not clear. In addition to triglycerides, chylomicrons carry fat-soluble vitamins like vitamin A and D, as well as bacterial lipopolysaccharide (LPS). ${ }^{17}$ The SI epithelium only weakly expresses most TLRs, ${ }^{18}$ and the most tolerogenic DC population $\left(\mathrm{CD} 103^{+} \mathrm{CD} 11 \mathrm{~b}^{-}\right)$within the lamina propria trafficking to the duodenal LN does not express TLR4 and other TLRs, with the exception of TLR9, ${ }^{7,19}$ potentially blunting the effect of LPS absorption within the proximal intestine.

Conversely, the proximal SI and draining lymph nodes absorb the highest concentrations of vitamin $A^{20-22}$ and its derivative, retinoic acid (RA). Importantly, RA exhibits a gradient along the intestine, and is likely responsible for multiple gut-specific immune features (see also GALT section below). Higher RA signaling in the $\mathrm{SI}$, evident by reporter studies, likely imprints $\mathrm{SI}$ migratory DCs to express higher levels of RALDH2, the enzyme responsible for converting vitamin $A$ to its immunomodulatory form, RA, compared to colonic DCs. ${ }^{20,22}$ RA is in part responsible for tolerogenic properties of DCs (see more under GALT below), and this difference between small intestinal versus colonic DCs is independent of the microbiota. ${ }^{20}$ Intriguingly, an evolutionarily conserved RA gradient along the gut also underlies the differentiation of the mid- versus hindgut during embryogenesis, 


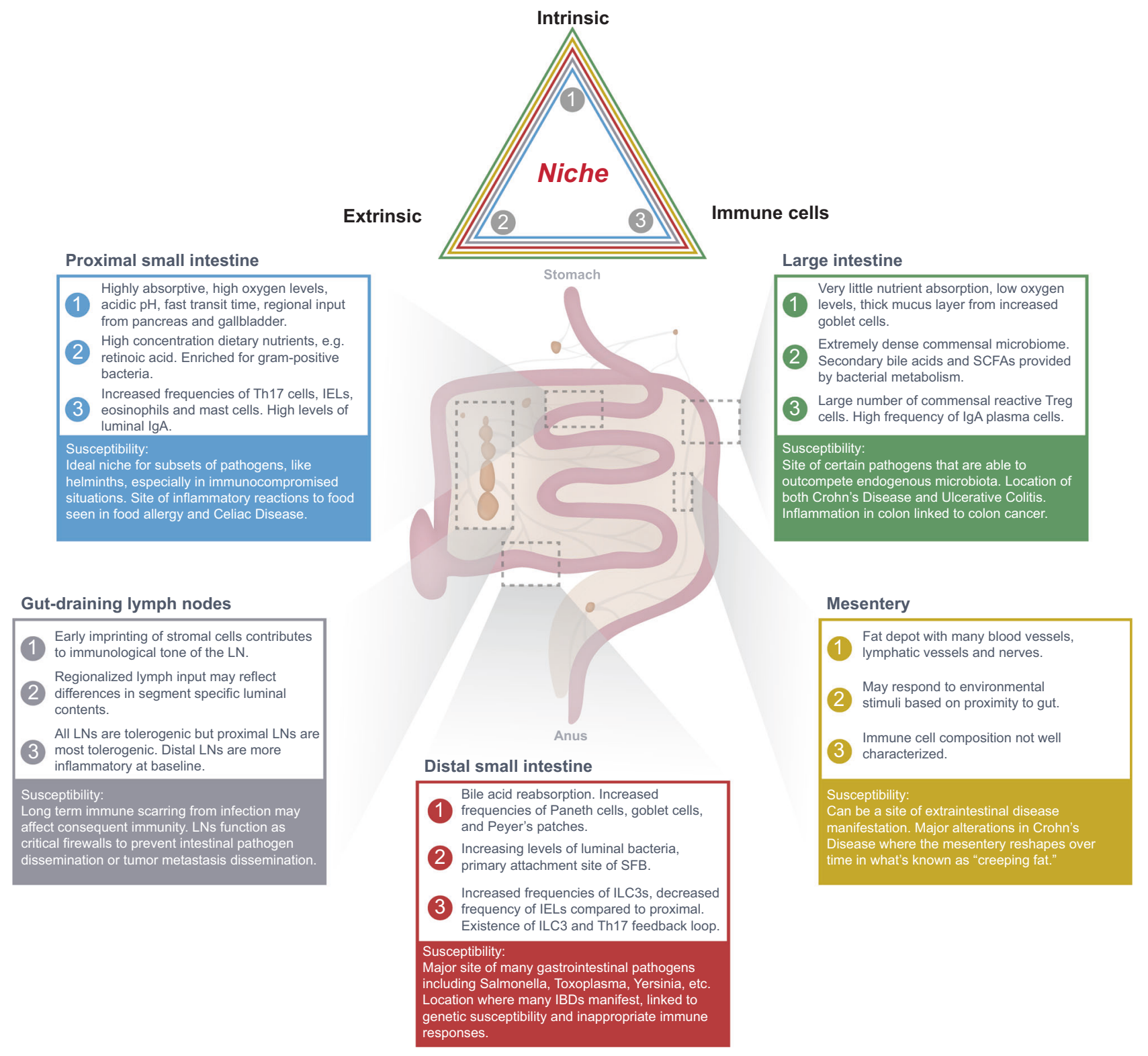

Fig. 1 Determinants of intestinal immune compartments. (Top middle) Each intestinal niche is influenced by a combination of three major factors: (1) intrinsic tissue properties, (2) extrinsic environmental signals, and (3) immune cell composition/immune propensities. To illustrate the unique synergy of these forces relative to each intestinal compartment, these factors have been examined for each location, as depicted by the middle figure, color coded by location. In addition, location specific infection and disease susceptibility is listed below each niche description. LN lymph node, IEL intraepithelial lymphocyte, SFB segmented filamentous bacteria, SCFAs short chain fatty acids.

suggesting that the tissue-intrinsic imprint of this factor on the duodenum dates back to its genesis. ${ }^{23}$

The absorptive functions of the proximal SI position this segment to be affected by dietary changes. Food variations, especially those that alter lipid or lipid-soluble vitamin content, such as high fat diets (HFD), may have a great impact here at the primary site of absorption. HFDs particularly associated with the "Western diet" have been implicated in many inflammatory conditions, ${ }^{24}$ though a full understanding of the consequences in the proximal $\mathrm{SI}$ is unknown. Additional food-derived compounds such as Aryl hydrocarbon receptor (AHR) ligands, found in cruciferous vegetables like broccoli, can have important immunological roles within the proximal SI. AHR, expressed by Th17, Tregs, ILCs, and IELs, is a major environmental sensor within the gut that responds to dietary, microbial and metabolic signals to initiate transcriptional programs. AHR expression is critical for maintaining the $\mathrm{CD} 8 \mathrm{aa}^{+}$IEL population within the SI, without which leads to an increase in bacterial load. ${ }^{25}$ Importantly, regulation of the downstream AHR pathway is critical in the survival of Th17 cells and ILC3s. ${ }^{26}$ Note, in many cases, dietary changes or deficiencies are accompanied by simultaneous effects on the resident microbiota, which may synergize to influence immune niches. ${ }^{27}$

Finally, the upper SI innate immune system appears skewed toward Type 2 rather than Type 1/17 responses, with epithelial correlates such as specialized Tuft cells, ${ }^{28}$ an enriched population of ILC2s that engages with Tuft cells upon infection, ${ }^{29}$ mast cells, and a remarkable presence of eosinophils ${ }^{1}$ that is also seen in germ free mice, ${ }^{30,31}$ potentially reflecting a co-evolution with helminths that have a preference for this niche (see more below). Collectively, the adaptations of the proximal SI immune system in response to the functional demands of nutrient absorption and pathogen exposure form the foundation of the immune niche. 
1262

Susceptibility to disease

The same reasons that restrict commensal fitness similarly limit infection within the upper SI. Nonetheless, some pathogens specifically thrive in the upper $\mathrm{SI}$, such as nematodes (e.g., $H$. Polygyrus, S. venezuelensis, N. brasiliensis $^{32}$ in rodents and A. lumbricoides, $S$. stercoralis in humans) and some protozoans such as Giardia lamblia. Clinically, patients with IgA deficiency are more prone to chronic giardiasis, highlighting the importance of duodenal $\lg A$ in protective immunity. ${ }^{33,34}$ In addition, some murine Gl viruses have been shown to also efficiently enter the host via the SI (rotaviruses, ${ }^{35}$ noroviruses, ${ }^{14,36-38}$ reoviruses ${ }^{39}$ ). Interestingly, in both mice and humans these viruses more successfully infect infants, potentially attributed to their immature immune system, waning of protective maternal antibodies or by default more "leaky" infant gut. ${ }^{40}$

Immunocompromising pre-conditions such as malnutrition, immunodeficiency, or the use of antibiotics lower the threshold for other serious $\mathrm{SI}$ infections such as $\mathrm{SI}$ bacterial overgrowth $\left(\mathrm{SIBO}^{41}\right)$, fungal infections ${ }^{42}$ or tropical sprue. ${ }^{43}$ These opportunistic pathogens can flourish in the otherwise nutrient rich environment of the upper SI. In most cases these infections damage the epithelium and lead to severe malabsorption, fatty stool, watery diarrhea or wasting syndromes in the case of long lasting infections.

The upper $\mathrm{SI}$ is also where Celiac disease and food allergies manifest (Box), whereby Celiac disease leads to auto-destructive epithelial damage, malabsorption and diarrhea, while food allergies are more life threatening due to the anaphylactic shock that can ensue. Though it seems evident that aversive reactions to food proteins take place in the Sl, where dietary components are highest in concentration, it is much less understood why they arise at all, given the immunosuppressed nature of this gut segment's draining LNs. ${ }^{44}$ Initial exposure to an antigen through the less tolerogenic $\mathrm{skin}^{45}$ or in the context of an infection are two nonmutually exclusive proposed mechanisms (see Box). For Celiac disease a genetic predisposition appears essential, ${ }^{46}$ yet only a small fraction of the population with susceptible haplotypes proceeds to disease. For food allergies a clear genetic link appears lacking, and so rather it is thought that environmental factors that render the immune system more pro-inflammatory toward food antigens play a decisive role for both diseases. ${ }^{4-49}$ Support for this theory comes from animal models in which infections of the SI were sufficient to trigger a loss of oral tolerance or food allergy development in otherwise wild type mice, ${ }^{8,20,50}$ as well as Celiac disease. ${ }^{39}$ In sum, these diseases appear specific to the SI precisely because it is the site of highest nutrient abundance.

\section{DISTAL SMALL INTESTINE}

Interplay between intrinsic and extrinsic factors

The distal SI, comprised mostly of the ileum, functions to absorb remaining nutrients missed by the proximal SI, mainly vitamin B12 and the reabsorption of nearly $95 \%$ of bile acids. Reflecting the lower nutrient absorption, the ileum possesses shorter villi and a thicker mucus layer compared to the proximal SI. However, increasing amounts of luminal bacteria, up to $10^{7} \mathrm{CFU}$ per $\mathrm{mL}$ necessitate increased epithelial barrier qualities such as increased frequencies of antimicrobial peptide secreting Paneth cells and mucus producing goblet cells.

Besides barrier maintenance, differences in non-hematopoietic cell frequencies of the epithelium may contribute to unique mechanisms of antigen sampling enriched within this intestinal segment. High frequencies of goblet cells within the distal SI expands the number of possible goblet cell-associated antigen passages (GAPs), which deliver luminal antigens to DCs within the lamina propria upon goblet cell secretion during homeostasis. ${ }^{51}$ Similarly, increased frequency and density of Peyer's patches (PPs) within the ileum may increase microfold cell (M-cell) mediated antigen uptake. These specialized epithelial cells reside in the follicle associated epithelium (FAE) where they capture and transport antigen to DCs present in the underlying subepithelial dome. Route of antigen entry, whether via cell mediated or cell free delivery to lymph nodes or inductive sites, can be highly consequential: For example, $M$ cell delivery induces an $\lg \mathrm{A}$ response in the underlying $B$ cell compartment, while direct antigen capture by DCs outside PPs triggers their migration to the draining lymph nodes, $T$ cell differentiation and help to preferentially non IgA-class switching, though $\lg A$ can also be induced in LNs. ${ }^{52}$ Antigen entry can also be modulated by the host such as the inhibition of GAPs during Salmonella infection, thereby limiting inflammation to innocuous luminal antigens. ${ }^{53}$ However, research querying consequences of antigen exposure is limited to very specific dietary or microbial antigens, and therefore much work is still needed to decipher how antigen characteristics and abundance, site of antigen exposure and cell type act in concert to shape adaptive immunity (see more under GALT).

Some distinct features of the ileum appear to be intrinsic to the tissue, such as the highest expression of not only bile acid transporters but also the transcription factor FXR in SI enterocytes. FXR-mediated bile acid signaling in ileum (and colon) elicits metabolic feedback adaptation to food intake, ${ }^{54}$ but also proinflammatory programs including IL-18 and iNOS, which contribute to limiting microbial growth. ${ }^{55}$ In addition, other epithelial cell types are enriched in the ileum, such as endocrine L-cells, implicated both in bile acid sensing via TGR5 and interaction with IELs.

Unlike the oxygen rich environment of the proximal SI, the ileum houses a large number of microbes, between $10^{3}-10^{7} \mathrm{CFU} /$ $\mathrm{mL}$, consisting of facultative and obligate anaerobes. Bacteroides and Clostridium species are common in this region of the intestine. Studies on prominent ileal species in rodents, segmented filamentous bacteria (SFB), have driven our understanding of tissue microenvironment influence on immunity over the last decade. SFB, anaerobic, unculturable bacteria, specifically colonize the ileum by attaching to the epithelium. Attachment, and transfer of antigen to epithelial cells results in the generation of SFB specific Th17 cells. ${ }^{4,56}$ Generation of Th17 cells is not exclusive to SFB and correlates with other attaching microbes. ${ }^{57,58}$ Whether there is a selective permissiveness for attachment and thereby a specific way of antigen uptake and epithelial stimulation in the ileum to support these Th17 responses, or if certain bacteria colonizing the ileum have evolved specific attaching mechanisms to their competitive advantage remains to be determined. Though Th17 cells are primed in the lymph nodes to express RORgt, only upon returning to the tissue will they secrete IL-17. Here, DCderived IL-23 drives ILC3 secretion of IL- $22^{59}$ to feed back onto the epithelium and induce Saa1/2,60 illustrating how cooperation between intestinal compartments can influence tissue specific immunity.

In addition to such specific direct interactions of bacteria with the ileal tissue, the microbiota also contributes to bioactive molecules in both the ileum and large intestine. These include microbial AHR ligands (see discussion in proximal $\mathrm{SI}$ ) and secondary bile acids, which have distinct immune signaling properties from primary bile acids, and TLR ligands (see more detail below in LI section).

\section{Susceptibility to disease}

The ileum is the Achilles heel of the SI when it comes to susceptibility to bacterial infection and pathogen-induced barrier breach, perhaps because it is on the one hand permissive of microbial growth and on the other of nutrient absorption. It also still has oxygen levels sustaining aerobic growth. As such, common food borne pathogens such as Toxoplasma, Salmonella, Vibrio Cholerae, Cryptosporidia, Listeria, and Yersinia grow in the ileum, and attach, enter and potentially disseminate through its 
epithelium, though each pathogen evolved a precise mechanism and target cell of infection. For example, $\mathrm{M}$ cells can be co-opted during infection by pathogens to gain entry beyond the epithelium as in the case of Salmonella. ${ }^{61}$ The main danger with all of these is potentially fatal systemic dissemination, though temporary disruption of epithelial function also leads to watery diarrhea.

The ileum is also a classical site for the manifestation of Crohn's disease (Box), an Inflammatory Bowel Disease (IBD) that can in principle occur along the entire digestive tract, with the ileocecal resection a frequent surgery. While the disease etiology of IBDs is incompletely understood, there is evidence that the combination of a genetic predisposition and an inappropriate pro-inflammatory immune response to the endogenous microbiota plays a major role. Notably, ileal Crohn's disease has the strongest genetic basis, and two disease susceptibility genes include $A T G 16 L 1^{62}$ and NOD2: ${ }^{63}$ Loss of function mutations in these genes lead to an inability of Paneth cells to mount antimicrobial responses, ${ }^{64}$ indicating both, that the ileal dependence on this cell type to keep the microbiota in check may make it vulnerable to this disease, and that outgrowth of pathogenic bacteria could be a consequence of such shift in host control. Again, the characteristics of the ileum, including highest density of bacteria and PPs, least suppression- prone draining LN within $\mathrm{SI}$, as well as selective absorptive capacity and nutrient signaling may contribute to this disease susceptibility.

\section{LARGE INTESTINE (LI)}

Interplay between intrinsic and extrinsic factors

By the time chyme reaches the LI, most nutrients have been absorbed in the SI, leaving only the small role of reabsorbing what water remains. Instead, the LI houses the bulk of microbes within the intestine with CFUs ranging up to $10^{12}$ bacteria per $\mathrm{mL}$. Without major nutrient absorptive requirements, evident by the lack of villi, the LI maintains a substantial barrier to preserve the integrity of the epithelium. To adequately sense and respond to the microbial load, strong expression of TLR4, TLR2, and TLR5 and increased numbers of mucus-secreting goblet cells mark the colonic epithelium. ${ }^{18}$ Despite their high numbers, colonic goblet cells do not form GAPs in the steady state due to sensing of luminal microbes. ${ }^{65}$ Instead they mainly serve to produce the thick mucus layer, separating the epithelium from luminal microbes. Other specialized cells like Paneth cells and IELs are rare compared to the SI. Luminal secretory $\lg \mathrm{A}$ is lower in the colon than SI, but IgA producing plasma cells are still abundant in its lamina propria and $\lg A$ is entangled in the outer mucus layer, fortifying the multilayered barrier of the LI.

The colon contains a high frequency of commensal-reactive, extrathymically generated suppressive Foxp $3^{+} \mathrm{CD} 4^{+}$T cells that are critical in immune homeostasis of the $\mathrm{LI}^{66} \mathrm{~A}$ dominant fraction of these Foxp $3^{+}$cells also express RORgt, generated in response to specific classes of commensal microbes. ${ }^{67,68}$ One notable example comes from Bacteroides fragilis which induces IL-10 production by Foxp $^{+}$cells through secretion of PSA. ${ }^{69}$ Interestingly, the colonic Foxp $3^{+} \mathrm{RORgt}^{+}$Treg cell frequency reaches an inheritable set point, determined early in life that cannot be exceeded even with microbial manipulation. ${ }^{70,71}$ Note that as opposed to mice, in humans, higher frequencies of Th17 cells are found in the colon while Th1 cells are more enriched in the distal colon, with no major differences in frequencies of Treg cells. ${ }^{72}$

High levels of secondary bile acids (BAs), biotransformed by certain bacterial species present in the colon have context dependent roles in influencing immune responses. For example, recent studies have implicated bile acids in skewing effector $T$ cell responses. Promotion of pTreg cells by the BA isoalloLCA is dependent on the conserved non-coding DNA sequence 3 enhancer of Foxp3 while the BA isoDCA increased Treg cell numbers via alterations to DCs. ${ }^{13,15}$ In contrast, in a model of high fat diet, certain bile acids promoted pathobiont outgrowth leading to the development of colitis in $1110^{-1-}$ mice. $^{73}$

Dietary changes that influence the LI often impart their effect through altering the short-chain fatty acid (SCFAs) production by the microbiota. Through the fermentation of mucus and dietary fiber, the colonic microbiota generates a number of immunomodulatory SCFAs such as butyrate, propionate and acetate. SCFAs are mostly restricted to the colon where the microbial load is highest, though through their absorption into the blood ${ }^{74}$ they may have wide ranging effects throughout the body. Butyrate for example has a number of immunomodulatory properties attributed to its HDAC inhibitory effect, including the reduction of proinflammatory cytokine production by $D C s^{74}$ and the expansion of IL-10 producing pTreg cells through action on the Foxp3 promoter, though direct effects on the protein and its translation have also been proposed. ${ }^{74,75}$ Pioneering work by Gordon and colleagues using defined consortia of bacteria has begun to experimentally explore the mechanisms by which diet modulates the microbiota, thus enabling more precise predictions about effects of diet. ${ }^{76,77}$

\section{Susceptibility to disease}

The $\mathrm{LI}$ is overall protected from damage by exogenous pathogens not only because the SI "sequesters them" before, but also due to its thick mucus layer and antimicrobial defenses. Nonetheless, some pathogens have optimized to thrive in the colon, usually by their ability to outcompete the endogenous microbiota with similar metabolic addictions combined with a mechanism to break the barrier. All are facultative or obligate anaerobes to survive under the colonic low oxygen conditions. Examples are Enterohemorrhagic Escherichia coli ${ }^{78}$ and Shigella flexneri. ${ }^{79}$ In addition, just like at the other sites, immunosuppression or use of antibiotics can lead to opportunistic colonic outgrowths of pathogens such as Clostridium difficile. ${ }^{80}$ In almost all cases, colonic infections lead to mucus rich and sometimes bloody diarrhea. Again, successful barrier breach can lead to fatality.

Given the high density of luminal bacteria, the colon like the terminal ileum is also a major site for IBD. However, in addition to Crohn's disease, here this also encompasses Ulcerative Colitis (UC, Box), which appears to be distinct in its etiology. As opposed to the patchy phenotype of Crohn's disease, UC is characterized by starting distally in the colon and progressively expanding to the more proximal colon. The genetic predispositions between UC and Crohn's disease only partially overlap ${ }^{81,82}$ and may explain the different sites of disease susceptibility along the intestine; in both cases the links that have been found include lowered innate defenses and immunosuppressive capacities, for example deficiencies in IL-10 and HNF4a in UC versus Paneth cell associated genes in Crohn's disease. ${ }^{83}$

Both IBDs increase the risk of colon cancer (Box), and the colon is the predominant site of malignancies in the intestine. It is not fully resolved if the chronic immunosuppressive treatment regime of IBD patients, a common genetic predisposition to both IBD and cancer, dysbiosis, or the chronic damage repair response in the epithelium make these patients susceptible to cancer. Mouse experiments suggest that the presence of the colonic microbiota in an inflamed intestine is critical for cancer development, as antibiotic-mediated elimination of bacteria or rendering mice entirely germ free substantially decreases colon cancer development. ${ }^{84}$ Thus, the need to turn over the epithelium in response to a microbiota (colon) rather than food absorption (small intestine) is linked to cancer.

IBDs are not the only risk factors for colon cancer, with the Metabolic Syndrome and age actually being the more common ones, though they cannot necessarily be uncoupled as people tend to get more obese in the course of a lifetime. Again, the reasons for cancer development in this setting are rather 
correlational, but include altered colonic microbiota, vasculature, and typically the consumption of a "Western Diet" that will not only change the microbiota but also impose a different metabolite composition affecting the colonic epithelium, e.g., increased levels of cancer promoting bile acids, secreted in response to dietary fat content and that are further modified by the microbiota. ${ }^{85,86}$

In addition, there are genetic predispositions for colon cancer, such as in Lynch syndrome, or hereditary non-polyposis colorectal cancer, associated with mutations in DNA mismatch repair genes family mutations, ${ }^{87}$ or familial adenomatous polyposis (FAP), where patients have inactivating mutations in adenomatous polyposis coli (APC gene), a negative regulator of beta catenin and thereby tumor suppressor. ${ }^{88}$ Loss of APC gene function is the most common mouse model of genetic intestinal cancer, but as opposed to humans, defective Apc in mice primarily leads to small bowel cancer. ${ }^{89}$ The fact that these mutations, which affect all cells in the intestine, primarily manifest in colorectal cancer may indicate that proliferation in the colon is under tighter control than in the small intestine, where the epithelium turns over rapidly always. The colonic environment may however also be more carcinogenic due to free radicals released from bacterial metabolism in a low oxygen environment. ${ }^{90}$

In sum, the colonic diseases reflect its bacterial niche and the demand to turn over tissue in high microbial presence.

\section{MESENTERY}

Interplay between intrinsic and extrinsic factors

The mesentery has long been documented to exist, but only recently has been designated a functional organ. This complex tissue consists of compartmentalized blood vessels, lymphatic vessels, and nerves in a spiderweb-like structure, whereby bundles of these routes connect each gut segment with the central arteries and veins, its draining LNs or the nervous system, respectively, and are surrounded by mesenteric adipose tissue (MAT). ${ }^{91}$ This mesenteric web also attaches the intestine to the abdominal wall, providing support to maintain proper positioning of the intestine for digestion. Sub-regions of the mesentery can be defined based on the associated intestinal segment, but functional differences according to sub-region are currently unknown. Similar to other adipose tissues, the MAT consists of a number of innate and adaptive immune cells, which can increase in frequency during colitis as a result of translocating bacteria, ${ }^{92}$ however the major functions of these cells in homeostasis and stress are not well understood.

The intimate association of the mesentery with the intestine positions this organ in an ideal location to respond to environmental stimuli from the gut. The mesentery is an important producer of C-reactive protein, especially in Crohn's disease, ${ }^{93}$ which can have important effects on systemic inflammation. Exactly how environmental signals, such as dietary or microbial changes in the intestine, are reflected in the mesentery is understudied. In models of colitis, bacterial translocation into the MAT is associated with MAT expression of cytokines and immune cell infiltration. ${ }^{92,94}$

\section{Susceptibility to disease}

The mesentery can be a site of extraintestinal disease manifestation. In an animal model of Yersinia infection, the pathogen leads to collecting lymphatic vessel damage in the mesentery, resulting in abdominal lymph leakage (chylous ascite), migration of dendritic cells into the mesenteric fat instead of their lymph node destination, and chronic inflammation of the mesenteric fat due to microbial leakage. ${ }^{8}$ As a result, adaptive immunity is changed from an immunosuppressive to pro-inflammatory state. In humans, the disease with the most apparent alteration in the mesentery is Crohn's disease, where this depot reshapes into what is perceived as "creeping fat". ${ }^{2,94,95}$ What causes this phenotype or what it translates to immunologically is poorly understood, but it is likely that lymphatic leakage and inflammation of the fat play a role. Also, it has recently been reported that tertiary lymphoid structures arise from the mesenteric lymphatic vessels of IBD patients, trapping $\mathrm{T}$ cells, B cells and $\mathrm{DCs} ;{ }^{96}$ what kind of immunological synapses can be formed and how they contribute to the etiology of advanced disease is currently still under investigation. The mesenteric fat depot also increases with obesity; while it has been largely ignored in the obesity field, which rather focuses on subcutaneous and non-mesenteric visceral fat, it is likely that it is inflamed under nutritional excess conditions as well and may contribute to the inflammatory state in the Metabolic Syndrome. Considering the mesentery as an immune compartment of the intestine in itself is relatively novel, and so the field currently lacks any insights into compartmental functions of the mesentery according to the gut segment it supports.

\section{GLNS AND GALT}

Interplay between intrinsic and extrinsic factors

In parallel to the gut, the inductive sites of immunity like the gLNs and GALT including the Peyer's patches and isolated lymphoid follicles (ILFs) display enormous heterogeneity. Despite their continuous appearance in mice, even the gLNs embedded in the mesentery, the mesenteric LNs ( $\mathrm{mLNs}$ ), receive lymph from distinct regions of gut and harbor unique immunological signatures dependent on location. Though all gLNs are considered immunosuppressive, in response to oral antigen, the LNs draining the proximal SI are biased to generate the most tolerogenic responses while the distal $\mathrm{SI}$ and colonic LNs are biased towards more inflammatory fates, consistent with differences in antigenpresenting cell (APC) gene expression profiles. ${ }^{20,97}$ Accordingly, tolerance to oral antigens is established in the proximal SI LNs, ${ }^{20,98}$ while colonic tolerance (albeit at lower pTreg inducing efficiencies, and distinct in nature as the cells co-express Rorgt) is established in the iliac and caudal LNs. ${ }^{99}$ Remarkably, these tolerogenic versus more inflammatory gradients from proximal to distal of LNs do not necessarily reflect the drained gut tissue properties, whereby the duodenum itself cannot be considered a particularly immunosuppressed environment and conversely, the colon harbors an extensive Treg pool.

Mechanistically, RA generated by DCs and stromal cells in proximal LNs regulates the TGF- $\beta$ driven differentiation of pTreg cells while inhibiting Th17 cell differentiation, ${ }^{100,101}$ but paradoxically is required for RORgt+ pTreg cells. ${ }^{68}$ As such, the induction of pTreg cells in response to oral antigen is highest in the duodenal lymph nodes where RA levels are high, while Th17 cell induction in response to this antigen is lowest. ${ }^{20}$ In addition, RA signaling during cell priming is responsible for the induction of the gut homing receptors $\alpha 4 \beta 7$ and CCR9 on $\mathrm{CD} 4^{+} \mathrm{T}^{\mathrm{T}}$ cells, CD8 ${ }^{+}$ $T$ cells and $B$ cells, ${ }^{102}$ allowing cells to traffic back to the $S I$ due to high CCL25 expression. It is important to note that despite its role in the promotion of Treg cells, low levels of vitamin A are also required for the generation of Type 1 and Type 17 responses in the context of other cytokines such as IL-15 (cytotoxic CD8 T cells) or IL-6 (Th17 cells), respectively, ${ }^{103}$ in agreement with the lower levels of Vitamin A and RALDH activity in the distal gLNs and their propensity to support more pro-inflammatory reactions in such cytokine contexts. ${ }^{101,104-106}$

Beyond RA availability, access to particular antigens differs along the gLNs (relative contribution of dietary versus microbial antigens and in vivo dose). While it is becoming increasingly apparent that the context in which an antigen is presented is the major driver for $\mathrm{T}$ cell fate decision, the dose in which an antigen is present may also matter in that high doses may make it into the blood, thereby leading to systemic antigen. This has formally been shown only for dietary antigen, ${ }^{107}$ though the critical role of gLNs and GALT in inducing intestinal T cell responses remain. ${ }^{108}$ Both 
dietary and microbial induced $\mathrm{T}$ cell responses may disseminate beyond the gut, as evidenced by the systemic nature of oral tolerance and conversely, the detrimental effect of SFB induced $\mathrm{T}$ cells on rheumatoid arthritis and experimental autoimmune encephalitis. $^{109,110}$

Other major LN dependent differences are reflected in the stromal cells that make up each $\mathrm{LN}^{111,112}$ suggesting the immunological tone in the LN may be a sum of inherent tissue properties and local lymph milieu as a result of gut segment specific content. Interestingly, the tolerogenic properties of $\mathrm{mLNs}$ are maintained after transplantation to the popliteal fossa in a DC dependent manner, underscoring the importance of stromal cells communication with DCs. ${ }^{111}$ Moreover, this stromal cell imprinting takes place during the neonatal period, setting the tolerogenic status of the mLNs early in life. ${ }^{112}$

In addition to draining intestinal segments, some gLNs co-drain multiple organs, most notably the pancreatic-duodenal LNs. Whether co-drainage with another organ impacts the baseline properties of these lymph nodes is not understood. This feature may be especially important in the more complicated human lymphatic system. Not only do humans have a far greater number of gLNs (well over 100), many are sequential in nature and several are connected to multiple organs. ${ }^{13}$ Thus, given our reliance on mouse models, our understanding of LN compartmentalization of immune responses is likely an underestimation of the complexity that exists in human immune responses.

As in the LNs, GALT displays location dependent differences in structure and function. Peyer's patches are present along the length of the SI but are heavily concentrated in the distal small intestine. They are characterized by continuous germinal center reactions (GCs), in SPF but not germ free mice, where the majority of SI IgA plasma cells are thought to be generated, ${ }^{114}$ though the gLNs are also capable of mounting IgA responses. In contrast, LI IgA plasma cells are generated in the cecal patches of the colon. ${ }^{115}$ This dichotomy is mirrored in humans where submucosal ILFs from the colon appear to give rise to colonic IgA plasma cells while the Peyer's patches were the main source of SI lgA. ${ }^{116}$ These differences are likely due to $\mathrm{SI}$ induction of a4 $\beta 7$ during priming versus GPR15 induction in the colon. Recent studies have uncovered previously unrecognized nuance to $\lg A$ with different mechanisms of maturation and modes of action when geared toward pathogens, specific members of the microbiota or broad classes of epitopes; ${ }^{17-119}$ however the manner in which these differ between the gut compartments is yet to be fully worked out.

\section{Susceptibility to disease}

In their role as sites of adaptive immune response initiation the gLNs are likely more involved in the human diseases along the intestine than currently appreciated. For example, in mice where the onset of diseases can be experimentally controlled, it has been shown that the duodenal LNs are the dominant site of food allergy initiation, while the colonic LNs are the main initiating site of adaptive immunity against the colonic pathogen Citrobacter. The gLNs can be substantially restructured by intestinal infections, in some cases long-term. Examples include lymphangiogenesis, swelling and a shift to a Th2 profile upon worm infections, lasting well beyond the period of worm clearance, and restructuring upon Yersinia infection. These post-infective immunological scars of the LNs may thus prospectively impact adaptive immune outcomes in the intestine. The gLNs also serve a critical "firewall" function against potentially disseminating microbiota and pathogens. ${ }^{120}$ In the context of Crohn's disease, LN granulomas are often found, indicating the leakage of bacteria. The interplay between adaptive functions of the LN and more innate pathogen containment efforts is not well studied. Finally, the gLNs are sites of colonic tumor metastasis dissemination, as this mechanism is implicated in virtually all metastatic cancers. As such, great care is taken to remove the pericolonic LNs during tumor surgery to investigate for metastasis. Since human gLNs are more complex in their organization, with converging LNs more distal to the gut, it is challenging to deliberately remove all LNs that drain a diseased segment of the intestine-but effort should likely be made both during IBD and cancer surgery to eliminate not only disseminated bacteria or cancer cells but also undesired immunological memory, to the extent it resides in the LNs and not tissue (see also Box).

\section{BROADER PHYSIOLOGICAL AND PATHOLOGICAL CONSEQUENCES OF REGIONALIZATION}

Compartmentalization of the intestinal immune system has broader physiological and pathophysiological consequences in addition to defining disease susceptibility and coping skills as discussed above. Beneficial consequences include the possibility to mount potentially antagonistic immune responses at the same time in a non-compromising way, such as inducing tolerance to food antigen in the SI while mounting a pro-inflammatory response to colonic Citrobacter infection. ${ }^{20}$ Site specific immune strategies also appear to have evolved such that they are compatible with the digestive function of each gut segment and focused toward the highest perils in a particular segment. For example, overall immunosuppression in the upper small intestine draining LNs prevents food allergy in this absorptive site, while the type2 immunity-prone profile of the gut tissue itself is equipped to expel helminths in their primary infection site. In a more neutral way, regionalization determines which nutrients can sustain particular beneficial immune cell functions, such as Vitamin A in the upper small intestine (pTreg cells, DCs), secondary bile acids in the ileum and large intestine (Th17 cells) or SCFAs in the colon (colonic Treg cells).

While we highlighted the distinctions between the compartments it is important to note that these boundaries are not absolute, and different compartments do communicate: In the most obvious way, anything in the duodenal lumen will eventually reach the colon, even if in a different form or concentration-but potentially sufficiently to stimulate the immune system. In humans the gLNs are not only much more numerous than in mice but also exist as sequential or converging nodes, making boundaries less strict. Furthermore, both in mice and men, some gLNs while exclusive to a gut niche, are shared with entirely different organs, potentially enabling direct inter-organ adaptive immune crosstalk. The most noteworthy examples are the pancreatic-duodenal lymph nodes, shared between duodenum and pancreas, and the common iliac lymph nodes, shared between the descending colon and pelvic organs such as urinary bladder, and reproductive tract. Finally, regionally instructed $B$ and $T$ cells recirculate and can thereby seed even extra-intestinal sites-though they will usually only expand upon re-encounter of antigen.

This "imperfect" compartmentalization of adaptive immunity has profound consequences, often to our benefit. The phenomenon of oral tolerance exemplifies this concept: it is thought that the circulation of immunosuppressive pTreg cells to food antigen generated in the SI gLNs prevents the allergic reaction to the same antigen encountered anywhere else in the body. ${ }^{121,122}$ Similarly, an intestinal pro-inflammatory adaptive immune response to a virus or immunogenic bacteria will contain their dissemination along the GI tract and even systemically. However, this very circulation of regionally shaped immune responses can also be detrimental. One widely studied example is the contribution of gut pathobiontreactive Th17 cells to autoimmune diseases such as rheumatoid arthritis (shown in mice ${ }^{110}$ and men $^{123}$ ) and Experimental Autoimmune Encephalitis, a mouse model for multiple sclerosis. ${ }^{109}$ While it is unclear what antigen these cells see in the periphery, both a bystander effect or sufficient bacterial antigen making it 


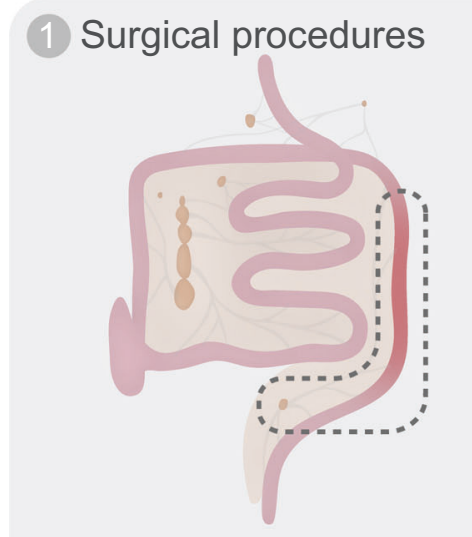

Removal of nearby lymph nodes during resectioning of the intestine for IBDs.

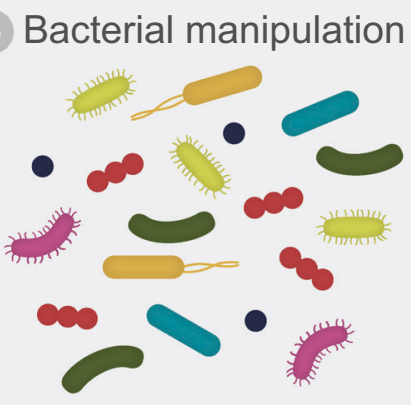

Synthetic communities
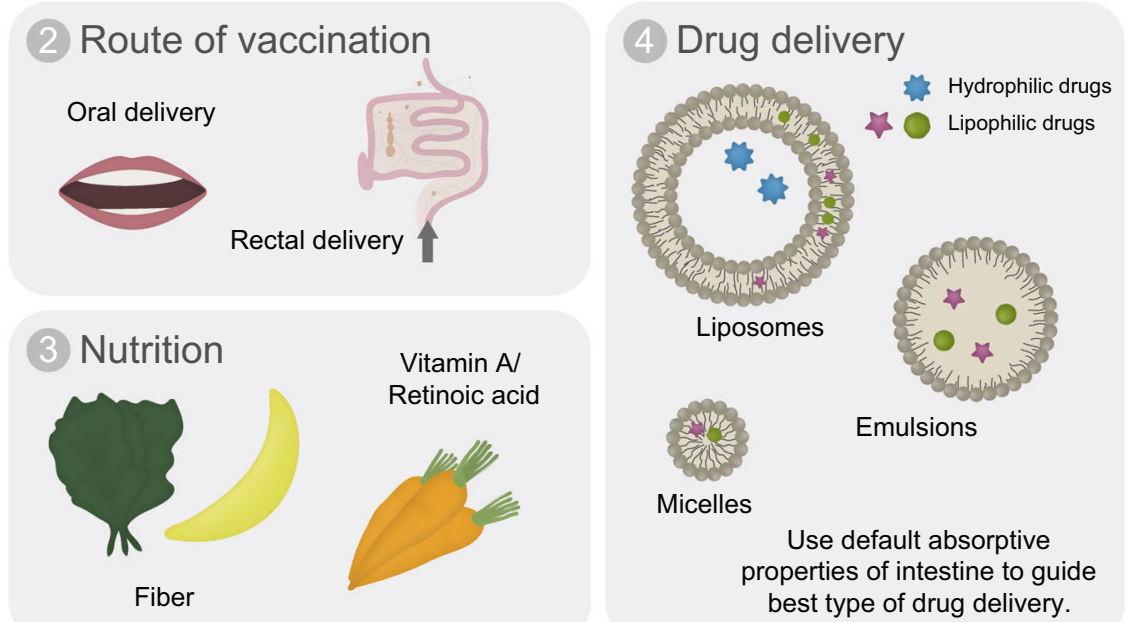

Emulsions

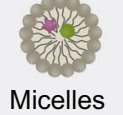

Micelles

Use default absorptive properties of intestine to guide best type of drug delivery.

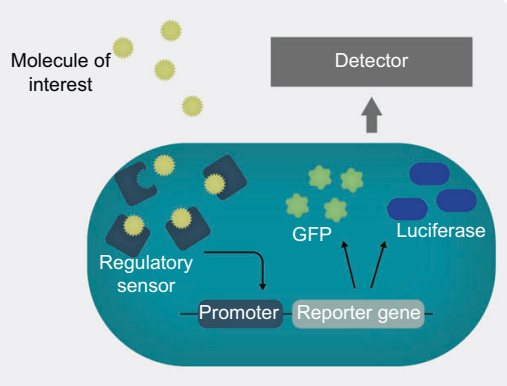

Bacteria as biosensors

Fig. 2 Regionalized implications for therapeutics. (1) Surgical procedures: During intestinal resection procedures for IBDs, removal of nearby LNs that harbor inflammatory lymphocytes may aid in preventing relapse of symptoms. (2) Route of vaccination: While there are some exceptions, oral vaccination strategies are often unsuccessful. This may be in part due to the immunosuppressive nature of the proximal small intestine. Other viable options for enteral vaccination may be through rectal delivery. (3) Nutrition: diets designed to alter or optimize microbial composition through different sources and amounts of fiber have therapeutic potential in intestinal disorders. Other dietary molecules like retinoic acid are critical for normal immune function, where deficiency leads to immunocompromising conditions. (4) Drug delivery: drugs that take advantage of the natural absorptive properties of the intestine may help regulate segment specific uptake of drugs. Lipid-soluble drugs may be more efficiently taken up in the proximal small intestine due to the high lipid absorption that takes place there. Liposomes, emulsions and micelles are different lipid based drug delivery options. (5) Bacterial manipulation: beyond just what bacteria are present in the gut these potential therapeutics focus on the behavior of the bacteria present. The generation of synthetic bacterial communities, composed of defined members that have been chosen to fulfill specific functions, is an exciting new avenue of microbiology. Furthermore, targeting bacteria through bacteriophages has the potential to alter the behavior of the resident microbiota to our benefit, or to simply deplete specific harmful bacteria. Last, bacteria can be utilized as living sensors of intestinal health, by genetically engineering novel sensing and reporting systems into the genome for external detection.

into the circulation have been proposed. It is also possible that redistribution along the gut of microbiota-reactive $T$ helper cells contributes to the spreading nature of Ulcerative Colitis (anal to proximal propagation of the disease) and Crohn's disease (patchy occurrence along the intestine but often starting at the ileo-cecal junction). Similarly, failure to mount pTreg cells to dietary antigen leads to systemic allergy such that e.g., topical encounter of peanut protein can lead to anaphylaxis. Last, an immune response in the gut could negatively influence adaptive immunity in a co-drained organ, such that e.g., co-drainage of duodenum and pancreas could promote the aggressiveness of pancreatic cancer or a duodenal infection trigger Type 1 Diabetes, a possibility we are investigating in our own lab.

The pathophysiological consequences of the way regionalization is set up are not confined to such situations with blurred boundaries of course. Rather, the regionally differential susceptibility to infection and disease makes the digestive tract overall vulnerable, as no one single evolutionary or therapeutic strategy will protect it while allowing for its metabolic functions.

\section{IMPLICATIONS FOR THERAPY AND TECHNOLOGY}

As we begin to appreciate the regional differences of the immune system, their connectivity and associated diseases we also start to reveal insights into current and future therapeutic interventions: both why certain therapies result in side effects or fail entirely and how to harness this knowledge to better tailor our approaches to the niche and the pathology (Fig. 2).

Strategies that have proven ineffective are for example oral vaccinations, which with few exceptions (e.g., Polio vaccine) fail to elicit protective immunity, even if the vaccine is shielded from digestive enzymes that certainly contribute to the challenge of the route. The immunosuppressive environment of the upper SI LNs may set a high threshold to overcome by adjuvant action of vaccines. Conversely, if enteral vaccine delivery is desired, then rectal delivery could at least immunologically be more effective. The potential for engineering approaches to modulate drug or vaccine delivery to distinct regions of the intestine could be enormously powerful in the future of gut therapeutics. In cases of pathology as is with IBD, surgical removal of inflamed segments of 
the intestine has been shown to cause only temporary alleviation of disease. While this is the standard care of treatment to prevent fatal rupture, in virtually all cases the disease reappears in a gut segment more proximal to the one removed. Intestinal resection alone not only leaves behind pro-inflammatory immunological memory, both in the lymph nodes that are not removed and redistributed $T$ cells, but re-adaptation of the intestinal epithelium to a novel function to adapt to the change may recreate a niche for dysbiotic bacteria. Efforts to remove all draining lymph nodes with the piece of intestine taken out in non-cancer surgery may help prevent such relapse.

In some cases, secondary pathologies result from standard treatments. For example, TNF inhibitors used to treat IBDs can promote systemic cancers due to the immunosuppressive nature, an effect not seen with IL-23 blockers. ${ }^{124}$ Similarly, anti CTLA4 and PD-1 based immune checkpoint inhibitors geared at other cancers in the body, can lead to severe colitis due to the unleashed proinflammatory program of both $\mathrm{CD}_{4}^{+}$and $\mathrm{CD} 8^{+} \mathrm{T}$ cells, often to the extent the therapy has to be stopped. ${ }^{125}$ Intriguingly, the microbial composition appears to play a role in whether this happens at all and in what severity, suggesting that engineering a protective microbial community to counter this periling side effect may be a way to reduce the risk. ${ }^{126,127}$ However, on the flip side, the efficacy of anti CTLA4 treatment is influenced by the microbiota, ${ }^{128}$ and the unintended killing of ileal epithelium in chemotherapy treatment of colon cancer appears to bolster a pro-inflammatory anti-tumor responses by virtue of invading bacteria. ${ }^{129}$

Finally, broad spectrum antibiotic treatment in the context of surgery or bone marrow transplant leads to susceptibility to GI infections such as Clostridium difficile due to its wipe-out of the intestinal microbiome. Here, fecal transplant has already proven an effective counter-measure, but the future may hold more deliberate selection of bacteria used to recolonize the gut.

Gnotobiotics and engineered therapeutic microbial communities are one way in which we can already start to apply our insights into the governing forces of intestinal immunity. These may not only be useful in the aforementioned contexts, rather it is hoped that every GI disease can be somewhat treated with a microbial approach. While still in their infancy, efforts to elicit beneficial immunological or metabolic effects by engineering bacterial genomes or altering bacterial behavior through bacteriophages or using bacteria as reporters for intestinal health are underway. Making use of our knowledge of which bacteria reside in what gut segment, but more importantly by what molecular mechanism will pave the way to also direct such communities to specific sites. Finding direct bacterial interactions with the gut epithelium through segment specific receptor-ligand pairs will be particularly attractive to achieve such targeting. Along the lines of microbe based approaches, our knowledge of what catabolic and anabolic metabolites specific members of the microbiota utilize is starting to pave a more informed way of food based therapy. ${ }^{77}$ Furthermore, our increasing understanding of which nutrients affect the immune system in what way along the intestine may yield nutritional therapeutic approaches independently of targeting the microbiome, such a dietary supplementation with Vitamin A to support tolerizing regimes to alleviate food allergy. The dietary context can also be used to target drugs to either the upper small intestine and the lymphatic route by virtue of lipid soluble drugs or micelles.

In sum, the fortunate combination of the microbiome research boom in recent years and our growing understanding of what governs the immune niches of the gut may pave the way for entirely new therapeutic strategies for restoring intestinal health.

\section{LIMITATIONS OF KNOWLEDGE}

While it is exciting to speculate on future site specific and personalized therapeutics these are limited by how much we really know about how the regional intestinal immune system is shaped and maintained. To this day, we do not fully understand the precise importance of diet, microbiome, virome and mycobiome and their interplay on host immunity, let alone in a spatially resolved manner. Part of the challenge is that host tissue, microbiome and dietary composition change not only in a circadian manner but also in the course of a lifetime, with critical time windows in perinatal and early childhood development ${ }^{130}$ and individual infection and medical treatment history impacting each aspect. Variation in genetic backgrounds of both humans and animal models add to the complexity of the assessment. Furthermore, some non-hematopoietic cell types have not yet been heavily investigated in their regional differences, such as the nervous system, stromal cells, blood and lymphatic vessels, all of which likely contribute to defining a milieu, potentially in a relatively hardwired manner.

Designing our studies such that they consider the temporal aspect rather than a snapshot of a situation is of course more costly and lengthy, but furthers our understanding of which components of a niche are temporary (e.g., due to fast cell turnover or transcriptional plasticity) versus permanent. Similarly, expanding the research to different genetic backgrounds in both mice and men will allow us to assess which factors are pan -vertebrate, and -human versus more individual determinants beyond clear disease associated SNPs.

\section{CONCLUSION}

In sum, the complexity of intrinsic and extrinsic driving forces shaping an intestinal niche makes it very challenging to determine causality in disease development and predicting effective therapeutic approaches, and we really only stand at the beginning of understanding this interplay. The joint effort of scientists from many different disciplines ranging from microbiology, immunology, metabolism, engineering, computation, basic, and translational approaches, their collaboration and easily accessible sharing of findings have helped make the progress we have made so far and will be even more crucial in the future to productively deal with the richness of information we obtain. These efforts will not only solve the mysteries of the alimentary tract but add to our understanding of what shapes tissue specific immunity in general, supporting fields well beyond mucosal immunology.

\section{Box- Major diseases of the intestine}

Celiac disease is a complex autoimmune disorder, in which the small intestinal epithelium is ultimately destroyed by cytolytic CD8+ T cells in response to dietary gluten. Other autoimmune features include the generation of antitransglutaminase 2 (TG2) antibodies and the requirement for specific HLA haplotypes, DQ2 or DQ8 in humans, with multiple additional susceptibility loci in pro-inflammatory cytokine genes. The sequence of events that lead to villous atrophy is still being elucidated, but the consensus so far is: Dietary gluten is deaminated by TG2, giving rise to negatively charged peptides that strongly bind to the MHCII DQ2 or DQ8 antigen pockets on DCs, and in conjunction with a pro-inflammatory profile DCs polarize gluten peptiderecognizing CD4+ T cells to a Th1 cell lineage. These T cells contribute to an inflammatory environment in the lamina propria and epithelium that activates cytolytic intraepithelial lymphocytes (IELs). In addition, gluten-specific CD4+ $T$ cells promote the generation of autoreactive anti-TG2 antibodies by providing help to TG2-specific B cells that have internalized TG2-gluten complexes and present gluten peptides. Evidence that there must be environmental triggers for the disease comes from the fact that only a fraction of people with the susceptible HLA haplotypes develop Celiac disease. These triggers play a role in rendering the DCs pro-inflammatory. They may also license the cytotoxic CD8 + T cells, which themselves are not recognizing gluten, to kill by upregulating the proinflammatory cytokine IL-15 and nonclassical MHC class I molecules that engage activating natural killer receptors expressed on IELs. Possible environmental triggers for innate immune activation are infections e.g., viruses ${ }^{39}$ but also inherent properties of the gluten peptide ${ }^{131}$ or non-gluten components of wheats. ${ }^{132}$ While the underlying factors that permitted the evolution of autoimmunity and the autoimmune-like reaction to a dietary antigen in Celiac disease are speculative, 
a positive correlation exists between historical wheat consumption and occurrence of the permissive HLA haplotypes. ${ }^{133}$ Treatment of Celiac disease is strict avoidance of gluten.

Food Allergy is a Type 2 immune response to particular ingested proteins. Th2 cells rather than pTreg cells recognizing food antigen are generated and mediate eosinophilia, mucus production and the generation of antigen specific IgE producing B cells that stimulate mast cell degranulation, which can lead to anaphylaxis. The reaction is reminiscent of anti-helminth responses. It is currently not clear what the context of type 2 prone priming is. Evidence exists for both a first exposure to the antigen through the $\mathrm{skin}^{45}$ or the intestine in an unfavorable context; ${ }^{134}$ both scenarios are not mutually exclusive. In practice the likelyhood of cutaneous versus intestinal antigen encounter may depend on the antigen. While genetic association with food allergy is poor, early life environmental factors such as exposure to microbes, type of birth and type of diet have been implicated. Intriguingly, food allergens can be categorized by features such as being structurally resistant to proteolytic cleavage, having protease inhibitory activity or binding lipids that may act as adjuvants ${ }^{135-137}$ all of which likely influence how they are presented to the immune system. Many plant allergens are also part of the germ line and so may have evolved to protect from being eaten by eliciting immune mediated food aversion. Still, in the majority of us they are not perceived as toxins that are to be expelled or avoided. Treatments for food allergy are elimination of the allergen from the diet or desensitization therapy by exposing individuals to small dose of the allergens, which can be sufficient to prevent anaphylactic shock. ${ }^{134}$

Crohn's disease is a progressive inflammatory bowel disease (IBD) in which anywhere along the Gl tract patches are inflamed and destroyed over time in intermittent "flares". The hallmark of Crohn's disease is the presence of granuloma. Common sites of initial manifestation however are the colon and the ileo-cecal junction, areas with high microbial density, and while inflammation starts in the epithelium it spreads transmurally and often into the mesentery. Critical to the inflammation is an uncontrolled pro-inflammatory T cell response (Th1 and pathogenic Th17 cells) to bacterial antigens; leading to diarrhea, bowel obstruction and in extreme cases perforation of the gut, necessitating surgical resection. First diagnosis can be as early as teenage years. Genetic predispositions include some that result in insufficient innate defenses of the epithelium, and those leading to an imbalance in Treg versus Th cell lineages, both of which may enable dysbiosis, favoring more pro-inflammatory bacterial outgrowth, higher susceptibility to infection and immune scarring after resolution. ${ }^{81}$ Currently, most common treatments of flares include antibiotics, thiopurines (aimed at rapidly dividing T cells), TNF (reducing proinflammatory cytokine action), $a 4 \beta 7$ antagonists (blocking $T$ cell trafficking to the intestine) and steroids (pleiotropic immunosuppressants), with several other treatment aimed specific cytokine pathways underway. ${ }^{138}$ Interestingly, while IL-23 antagonism appears like an efficient anti-inflammatory new strategy, ${ }^{139}$ IL-17 blockade aggravates the disease, suggesting that the enrichment of Th17 cells is in its core a tissue repair mechanism in this context. ${ }^{140,141}$ None are curative.

Ulcerative Colitis is a colonic IBD that starts at the rectum but can spread progressively all the way up to the cecum. It manifests as chronically inflamed ulcers in the epithelium that lead to colonic dysfunction, with the typical age of onset in early adulthood. While the genetic predispositions only partially overlap with those of Crohn's disease (see text), it is thought that an inappropriate proinflammatory reaction to the microbiota also underlies the disease, and the same biologics as for Crohn's disease are being used to treat ulcerative colitis, with different levels of efficacy. However, as opposed to Crohn's disease surgical removal of a diseased gut segment is usually curative, and while there are many possibilities to the underlying reason one is that colonic surgery includes the removal of the pericolonic lymph nodes, while in small intestinal surgery that is typically needed in Crohn's disease surgery the lymph nodes largely stay behind due to their more gut-remote anatomy.

Colon Cancers malignancies arise from uncontrolled proliferation and dedifferentiation of colonic epithelial cells. Sporadic colorectal cancers typically start as polyps that can turn malignant, while IBD-associated cancer starts as flat lesions. Environmental stress factors that prompt epithelial repair mechanisms are thought to trigger colorectal cancer and include IBDs, obesity, microbial shifts, alcohol consumption, smoking and age. More rare, genetic predispositions also exist (see text).

\section{AUTHOR CONTRIBUTIONS}

H.B. and D.E conceived the manuscript and figures and wrote the manuscript. H.B. designed the figures.

\section{FUNDING}

Support: D.E. is supported by the University of Chicago, a University of Chicago NIDDK- P\&F grant and DRTC P\&F grant, the Pew Charitable Trust, the Searle Scholars Program, The Cancer Research Foundation and the Pancreatic Cancer Action Network.

\section{ADDITIONAL INFORMATION}

Competing interests: The authors declare no competing interests.

Publisher's note Springer Nature remains neutral with regard to jurisdictional claims in published maps and institutional affiliations.

\section{REFERENCES}

1. Mowat, A. M. \& Agace, W. W. Regional specialization within the intestinal immune system. Nat. Rev. Immunol. 14, 667-685 (2014).

2. Agace, W. W. \& McCoy, K. D. (2017). Regionalized development and maintenance of the intestinal adaptive immune landscape. Immunity. 46, 532-548, https://doi.org/10.1016/j.immuni.2017.04.004

3. Sujino, T., et al (2016). Tissue adaptation of regulatory and intraepithelial CD4+ T cells controls gut inflammation. Science. https://doi.org/10.1126/science.aaf3892

4. Ivanov, I. I. et al. Induction of intestinal Th17 cells by segmented filamentous bacteria. Cell 139, 485-498 (2009).

5. Hapfelmeier, S., et al (2010). Reversible microbial colonization of germ-free mice reveals the dynamics of IgA immune responses. Science. https://doi.org/10.1126/ science. 1188454

6. Cerovic, V., et al (2013). Intestinal CD103- dendritic cells migrate in lymph and prime effector T cells. Mucosal Immunology. https://doi.org/10.1038/mi.2012.53

7. Esterházy, D. et al. Classical dendritic cells are required for dietary antigen-mediated induction of peripheral Treg cells and tolerance. Nat. Immunol. 17, 505-513 (2016).

8. Fonseca, D. M. et al. Microbiota-Dependent Sequelae of Acute Infection Compromise Tissue-Specific. Immun. Cell 163, 354-366 (2015).

9. Bunker, J. J. et al. Innate and Adaptive Humoral Responses Coat Distinct Commensal Bacteria with Immunoglobulin A. Immunity 43, 541-553 (2015).

10. Reynoso-Paz, S., et al (1999). The immunobiology of bile and biliary epithelium. Hepatology. https://doi.org/10.1002/hep.510300218

11. Donaldson, G. P., Lee, S. M. \& Mazmanian, S. K. Gut biogeography of the bacterial microbiota. Nat. Publ. Group 14, 1-13 (2015).

12. Saffouri, G. B., et al (2019). Small intestinal microbial dysbiosis underlies symptoms associated with functional gastrointestinal disorders. Nat. Commun. 10, 2012. https://doi.org/10.1038/s41467-019-09964-7

13. Campbell, C. et al. Bacterial metabolism of bile acids promotes generation of peripheral regulatory T cells. Nature 581, 1-21 (2020).

14. Grau, K. R. et al. The intestinal regionalization of acute norovirus infection is regulated by the microbiota via bile acid-mediated priming of type III interferon. Nat. Microbiol. 5, 84-92 (2020).

15. Hang, S., et al (2020). Bile acid metabolites control TH17 and Treg cell differentiation. Nature, 1-25. https://doi.org/10.1038/s41586-019-1785-z

16. Bernier-Latmani, J. \& Petrova, T. V. Intestinal lymphatic vasculature: structure, mechanisms and functions. Nat. Rev. Gastroenterol. Hepatol. 14, 510-526 (2017).

17. Ghoshal, S., Witta, J., Zhong, J., de Villiers, W. \& Eckhardt, E. Chylomicrons promote intestinal absorption of lipopolysaccharides. J. Lipid Res. 50, 90-97 (2009).

18. Price, A. E. et al. A map of toll-like receptor expression in the intestinal epithelium reveals distinct spatial, cell type-specific, and temporal patterns. Immunity 49, 560-575.e6 (2018).

19. Cerovic, Vuk et al. Hyporesponsiveness of intestinal dendritic cells to TLR stimulation is limited to TLR4. J. Immunol. 182, 2405-2415 (2009).

20. Esterházy, D. et al. Compartmentalized gut lymph node drainage dictates adaptive immune responses. Nature 569, 126-130 (2019).

21. Goncalves, A. L. et al. Fat-soluble vitamin intestinal absorption: absorption sites in the intestine and interactions for absorption. Food Chem. 172, 155-160, https://doi.org/10.1016/j.foodchem.2014.09.021 (2015).

22. Jaensson-Gyllenbäck, E., et al (2019). Bile retinoids imprint intestinal CD103+ dendritic cells with the ability to generate gut-tropic T cells. Mucosal Immunol., 1-10. about:client

23. Hou, J., et al (2014). A regulatory network controls nephrocan expression and midgut patterning. Development (Cambridge). https://doi.org/10.1242/ dev.108274

24. Christ, A., Lauterbach, M. \& Latz, E. Western diet and the immune system: an inflammatory connection. Immunity 51, 794-811 (2019).

25. $\mathrm{Li}, \mathrm{Y}$. et al. Exogenous stimuli maintain intraepithelial lymphocytes via aryl hydrocarbon receptor activation. Cell 147, 629-640 (2011).

26. Schiering, C. et al. Feedback control of AHR signalling regulates intestinal immunity. Nature 542, 242-245 (2017).

27. Subramanian, S., et al (2014). Persistent gut microbiota immaturity in malnourished Bangladeshi children. Nature. https://doi.org/10.1038/nature13421 
28. Schütz, B. et al. Distribution pattern and molecular signature of cholinergic tuft cells in human gastro-intestinal and pancreatic-biliary tract. Sci. Rep. 9, 17466 (2019).

29. Schneider, C., et al (2018). A metabolite-triggered tuft Cell-ILC2 circuit drives small intestinal remodeling. Cell, 174. https://doi.org/10.1016/j.cell.2018.05.014

30. Chu, D. K., et al (2014). Indigenous enteric eosinophils control DCs to initiate a primary Th2 immune response in vivo. J. Exp. Med. https://doi.org/10.1084/ jem. 20131800

31. Jiménez-Saiz, R., et al (2020). Microbial regulation of enteric eosinophils and its impact on tissue remodeling and Th2 immunity. Front. Immunol. https://doi.org/ 10.3389/fimmu.2020.00155

32. Marshall, J. S., Wells, P. D. \& Bell, E. B. Accelerated elimination of N. brasiliensis from the small intestine after auto-anti-lgE induction. Immunology. 60, 303-308 (1987).

33. Yazdani, R., Azizi, G., Abolhassani, H., \& Aghamohammadi, A. (2017). Selective IgA deficiency: epidemiology, pathogenesis, clinical phenotype, diagnosis, prognosis and management. In Scand. J. Immunol. https://doi.org/10.1111/ sji.12499

34. Zinneman, H. H., \& Kaplan, A. P. (1972). The association of giardiasis with reduced intestinal secretory immunoglobulin A. Am. J. Digest. Dis. https://doi. org/10.1007/BF02231148

35. Zhu, S., et al (2017). Nlrp9b inflammasome restricts rotavirus infection in intestinal epithelial cells. Nature. https://doi.org/10.1038/nature22967

36. Troeger, $\mathrm{H}_{\text {., }}$ et al (2009). Structural and functional changes of the duodenum in human norovirus infection. Gut. https://doi.org/10.1136/gut.2008.160150

37. Wilen, C. B. et al. Tropism for tuft cells determines immune promotion of norovirus pathogenesis. Science 360, 204-208 (2018).

38. Karst, S. M., \& Wobus, C. E. (2015). A Working Model of How Noroviruses Infect the Intestine. PLoS Pathogens. https://doi.org/10.1371/journal.ppat.1004626

39. Bouziat, R., et al (2017). Reovirus infection triggers inflammatory responses to dietary antigens and development of celiac disease. Science, 356.

40. Catassi, C., Bonucci, A., Coppa, G. V., Carlucci, A., \& Giorgi, P. L. (1995). Intestinal permeability changes during the first month: Effect of natural versus artificial feeding. J. Pediatric Gastroenterol. Nutr. https://doi.org/10.1097/00005176199511000-00003

41. Ponziani, F. R., Gerardi, V., \& Gasbarrini, A. (2016). Diagnosis and treatment of small intestinal bacterial overgrowth. Expert Rev. Gastroenterol. Hepatol. https:// doi.org/10.1586/17474124.2016.1110017

42. Erdogan, A., \& Rao, S. S. C. (2015). Small intestinal fungal overgrowth. In Current Gastroenterology Reports. https://doi.org/10.1007/s11894-015-0436-2

43. Brown, I. S., Bettington, A., Bettington, M., \& Rosty, C. (2014). Tropical sprue: Revisiting an underrecognized disease. Am. J. Surg. Pathol. https://doi.org/ 10.1097/PAS.0000000000000153

44. Kim, K. S. et al. Dietary antigens limit mucosal immunity by inducing regulatory T cells in the small intestine. Science 351, 858-863 (2016).

45. Tordesillas, L., et al (2014). Skin exposure promotes a Th2-dependent sensitization to peanut allergens. Journal of Clinical Investigation, 124. https://doi.org/ $10.1172 / J C I 75660$

46. Sollid, L. M. (2000). Molecular basis of celiac disease. Ann. Rev. Immunol. https:// doi.org/10.1146/annurev.immunol.18.1.53

47. Blázquez, A. B., \& Berin, M. C. (2017). Microbiome and food allergy. In Transl. Res. https://doi.org/10.1016/j.trsl.2016.09.003

48. Caminero, A., Meisel, M., Jabri, B., \& Verdu, E. F. (2019). Mechanisms by which gut microorganisms influence food sensitivities. Nat. Rev. Gastroenterol. Hepatol. https://doi.org/10.1038/s41575-018-0064-z

49. Cheng, J. et al. Duodenal microbiota composition and mucosal homeostasis in pediatric celiac disease. BMC Gastroenterol. 13, 113 (2013).

50. Bouziat, R. et al. Murine norovirus infection induces $\mathrm{TH} 1$ inflammatory responses to dietary antigens. Cell Host Microbe 24, 677-688.e5 (2018).

51. McDole, J. R. et al. Goblet cells deliver luminal antigen to CD103+ dendritic cells in the small intestine. Nature 483, 1-6 (2012).

52. Rios, D., et al (2016). Antigen sampling by intestinal M cells is the principal pathway initiating mucosal $\lg \mathrm{A}$ production to commensal enteric bacteria Mucosal Immunol. https://doi.org/10.1038/mi.2015.121

53. Kulkarni, D. H., et al (2018). Goblet cell associated antigen passages are inhibited during Salmonella typhimurium infection to prevent pathogen dissemination and limit responses to dietary antigens. Mucosal Immunol., 1-11. https://doi.org/ 10.1038/s41385-018-0007-6

54. Houten, S. M., Volle, D. H., Cummins, C. L., Mangelsdorf, D. J., \& Auwerx, J. (2007). In vivo imaging of farnesoid $X$ receptor activity reveals the ileum as the primary bile acid signaling tissue. Mol. Endocrinol. https://doi.org/10.1210/me.2007-0113

55. Inagaki, T., et al (2006). Regulation of antibacterial defense in the small intestine by the nuclear bile acid receptor. Proc. Natl. Acad. Sci. USA. https://doi.org/ 10.1073/pnas.0509592103
56. Ladinsky, M. S. et al. Endocytosis of commensal antigens by intestinal epithelial cells regulates mucosal T cell homeostasis. Science 363, eaat4042 (2019).

57. Tan, T. G. et al. Identifying species of symbiont bacteria from the human gut that, alone, can induce intestinal Th17 cells in mice. Proc. Natl. Acad. Sci. 113, E8141 LP-E8150, https://doi.org/10.1073/pnas.1617460113 (2016).

58. Atarashi, K. et al. Th17 cell induction by adhesion of microbes to intestinal epithelial cells. Cell 163, 1-38 (2016).

59. Eken, A., Singh, A. K., Treuting, P. M., \& Oukka, M. (2014). IL-23R+ innate lymphoid cells induce colitis via interleukin-22-dependent mechanism. Mucosal Immunol., 7. https://doi.org/10.1038/mi.2013.33

60. Sano, T. et al. An IL-23R/IL-22 circuit regulates epithelial serum amyloid $A$ to promote local effector Th17 responses. Cell 163, 381-393 (2015).

61. Jones, B. D., Ghori, N. \& Falkow, S. Salmonella typhimurium initiates murine infection by penetrating and destroying the specialized epithelial $\mathrm{M}$ cells of the Peyer's patches. J. Exp. Med. 180, 15-23 (1994).

62. Lala, S., et al (2003). Crohn's disease and the NOD2 gene: a role for paneth cells Gastroenterology. https://doi.org/10.1016/S0016-5085(03)00661-9

63. Ahmad, T., et al (2002). The molecular classification of the clinical manifestations of Crohn's disease. Gastroenterology. https://doi.org/10.1053/gast.2002.32413

64. Cadwell, K., et al (2008). A key role for autophagy and the autophagy gene Atg16l1 in mouse and human intestinal Paneth cells. Nature. https://doi.org/ 10.1038 /nature07416

65. Knoop, K. A. et al. Microbial antigen encounter during a preweaning interval is critical for tolerance to gut bacteria. Sci. Immunol. 2, eaao1314-24 (2017).

66. Atarashi, K. et al. Induction of colonic regulatory T cells by indigenous Clostridium species. Science 331, 337-341 (2011).

67. Sefik, E. et al. Individual intestinal symbionts induce a distinct population of RORY ${ }^{+}$regulatory T cells. Science 349, 993-997 (2015).

68. Ohnmacht, C. et al. The microbiota regulates type 2 immunity through RORyt + T cells. Science 349, 1-9 (2015).

69. Round, J. L. \& Mazmanian, S. K. Inducible Foxp3 $3^{+}$regulatory T-cell development by a commensal bacterium of the intestinal microbiota. Proc. Natl Acad. Sci. 107, 12204 LP-12209 (2010).

70. Ramanan, D., et al (2020). An immunologic mode of multigenerational transmission governs a Gut Treg Setpoint. Cell, 1-29. https://doi.org/10.1016/j. cell.2020.04.030

71. Geva-Zatorsky, N. et al. Mining the human Gut microbiota for immunomodulatory organisms. Cell 168, 928-943.e11 (2017).

72. James, K. R. et al. Distinct microbial and immune niches of the human colon Nat. Immunol. 21, 343-353 (2020).

73. Devkota, S. et al. Dietary-fat-induced taurocholic acid promotes pathobiont expansion and colitis in II10-/- mice. Nature 487, 104-108 (2012).

74. Arpaia, N., et al (2013). Metabolites produced by commensal bacteria promote peripheral regulatory T-cell generation. Nature, 504. https://doi.org/10.1038/ nature12726

75. Furusawa, Y. et al. Commensal microbe-derived butyrate induces the differentiation of colonic regulatory T cells. Nature 504, 446-450 (2013).

76. Faith, J. J., McNulty, N. P., Rey, F. E. \& Science, J. I. G. Predicting a human gut microbiota's response to diet in gnotobiotic mice. Science 333, 97-101 (2011). \& 2011.

77. Patnode, M. L. et al. Interspecies Competition Impacts Targeted Manipulation of Human Gut Bacteria by Fiber- Derived Glycans. Cell 179, 59-73.e13 (2019)

78. Woodward, S. E., Krekhno, Z., \& Finlay, B. B. (2019). Here, there, and everywhere: How pathogenic Escherichia coli sense and respond to gastrointestinal biogeography. Cell. Microbiol. https://doi.org/10.1111/cmi.13107

79. Philpott, D. J., Edgeworth, J. D., \& Sansonetti, P. J. (2000). The pathogenesis of Shigella flexneri infection: Lessons from in vitro and in vivo studies. Philos. Trans. R. Soc. B: Biological Sci. https://doi.org/10.1098/rstb.2000.0599

80. Buffie, C. G. et al. Precision microbiome reconstitution restores bile acid mediated resistance to Clostridium difficile. Nature 517, 205-208 (2014).

81. Ek, W. E., D'Amato, M., \& Halfvarson, J. (2014). The history of genetics in inflammatory bowel disease. Ann. Gastroenterol.

82. Khor, B., Gardet, A., \& Xavier, R. J. (2011). Genetics and pathogenesis of inflammatory bowel disease. Nature. https://doi.org/10.1038/nature10209

83. Chellappa, K., et al (2016). Opposing roles of nuclear receptor HNF4a isoforms in colitis and colitis-associated colon cancer. ELife. https://doi.org/10.7554/ elife.10903

84. Leystra, A. A. \& Clapper, M. L. (2019). Gut microbiota influences experimental outcomes in mouse models of colorectal cancer. Genes. https://doi.org/10.3390/ genes 10110900

85. Van Kruiningen, H. J. \& Colombel, J.-F. The forgotten role of lymphangitis in Crohn's disease. Gut 57, 1-4 (2008).

86. Wan, Y., et al (2020). Unconjugated and secondary bile acid profiles in response to higher-fat, lower-carbohydrate diet and associated with related gut 
microbiota: A 6-month randomized controlled-feeding trial. Clin. Nutr. https:// doi.org/10.1016/j.clnu.2019.02.037

87. Sehgal, R., et al (2014). Lynch syndrome: an updated review. Genes. https://doi. org/10.3390/genes5030497

88. Rustgi, A. K. (2007). The genetics of hereditary colon cancer. Genes Dev. https:// doi.org/10.1101/gad.1593107

89. Taketo, M. M. (2006). Mouse models of gastrointestinal tumors. Cancer Sci. 97. https://doi.org/10.1111/j.1349-7006.2006.00190.x

90. Babbs, C. F. (1990). Free radicals and the etiology of colon cancer. Free Radical Biol Med. https://doi.org/10.1016/0891-5849(90)90091-V

91. Coffey, J. C. \& O'Leary, D. P. The mesentery: structure, function, and role in disease. Lancet Gastroenterol. Hepatol. 1, 238-247 (2016).

92. Batra, A. et al. Mesenteric fat-control site for bacterial translocation in colitis? Mucosal Immunol. 5, 580-591 (2012).

93. Peyrin-Biroulet, L. et al. Mesenteric fat as a source of $C$ reactive protein and as a target for bacterial translocation in Crohn's disease. Gut 61, 78-85 (2012).

94. Ha, C. W. Y. et al. Translocation of Viable Gut Microbiota to Mesenteric Adipose Drives Formation of Creeping Fat in Humans. Cell 183, 666-683.e17 (2020).

95. Mao, R., et al (2019). The mesenteric fat and intestinal muscle interface: creeping fat influencing stricture formation in Crohn's disease. Inflamm. Bowel Dis. https:// doi.org/10.1093/ibd/izy331

96. McNamee, E. N., \& Rivera-Nieves, J. (2016). Ectopic tertiary lymphoid tissue in inflammatory bowel disease: protective or provocateur? Front. Immunol. https:// doi.org/10.3389/fimmu.2016.00308

97. Houston, S. A. et al. The lymph nodes draining the small intestine and colon are anatomically separate and immunologically distinct. Mucosal Immunol. 9, 468-478 (2016).

98. Spahn, T. W. et al. Mesenteric lymph nodes are critical for the induction of highdose oral tolerance in the absence of Peyer's patches. Eur. J. Immunol. 32, 1109-1113 (2002).

99. Veenbergen, S. et al. Colonic tolerance develops in the iliac lymph nodes and can be established independent of $\mathrm{CD}_{103^{+}}$dendritic cells. Mucosal Immunol. 9, 894-906 (2016).

100. Bettelli, E. et al. Reciprocal developmental pathways for the generation of pathogenic effector TH17 and regulatory T cells. Nature 441, 235-238 (2006).

101. Mucida, D. et al. Reciprocal TH17 and regulatory T cell differentiation mediated by retinoic acid. Science 317, 256-260 (2007).

102. Mora, J. R. et al. Selective imprinting of gut-homing T cells by Peyer's patch dendritic cells. Nature 424, 88-93 (2003).

103. Bono, M. R., et al (2016). Retinoic acid as a modulator of $\mathrm{T}$ cell immunity. Nutrients 8. https://doi.org/10.3390/nu8060349

104. Takahashi, $H_{\text {., }}$ et al (2012). TGF- $\beta$ and retinoic acid induce the microRNA miR$10 \mathrm{a}$, which targets $\mathrm{Bcl}-6$ and constrains the plasticity of helper T cells. Nat. Immunol. 13. https://doi.org/10.1038/ni.2286

105. Hall, J. A. et al. Essential role for retinoic acid in the promotion of CD4+ T cell effector responses via retinoic acid receptor alpha. Immunity 34, 435-447 (2011).

106. Jabri, B. \& Abadie, V. (2015). IL-15 functions as a danger signal to regulate tissueresident $\mathrm{T}$ cells and tissue destruction. In Nat. Rev Immunol. https://doi.org/ 10.1038/nri3919

107. Pabst, O. \& Mowat, A. M. Oral tolerance to food protein. Mucosal Immunol. 5, 232-239 (2012).

108. Hadis, U. et al. Intestinal tolerance requires gut homing and expansion of FoxP3+ regulatory T cells in the Lamina Propria. Immunity 34, 237-246 (2011).

109. Lee, Y. K., Menezes, J. S., Umesaki, Y. \& Mazmanian, S. K. Proinflammatory T-cell responses to gut microbiota promote experimental autoimmune encephalomyelitis. Proc. Natl Acad. Sci. 108, 4615-4622 (2011).

110. $\mathrm{Wu}, \mathrm{H}$. J. et al. Gut-residing segmented filamentous bacteria drive autoimmune arthritis via T helper 17 cells. Immunity 32, 815-827 (2010).

111. Cording, S. et al. The intestinal micro-environment imprints stromal cells to promote efficient Treg induction in gut-draining lymph nodes. Mucosal Immunol. 7, 359-368 (2014).

112. Pezoldt, J., et al (2018). Neonatally imprinted stromal cell subsets induce tolerogenic dendritic cells in mesenteric lymph nodes. Nat. Commun., 1-14. https://doi.org/10.1038/s41467-018-06423-7

113. Gray, H. Anatomy of the Human Body. Philadelphia: Lea \& Febiger, 1918; Bartleby.com, 2000. www.bartleby.com/107/.

114. Craig, S. W., \& Cebra, J. J. (1971). Peyer's patches: An enriched source of precursors for iga-producing immunocytes in the rabbit. J. Exp. Med., 134. https:// doi.org/10.1084/jem.134.1.188

115. Masahata, K. et al. Generation of colonic lgA-secreting cells in the caecal patch. Nat. Commun. 5, 3704 (2014).
116. Fenton, T. M. et al. Immune profiling of human gut-associated lymphoid tissue identifies a role for isolated lymphoid follicles in priming of region-specific immunity. Immunity 52, 1-21 (2020).

117. Rogier, E. W., Frantz, A. L., Bruno, M. E. C., \& Kaetzel, C. S. (2014). Secretory lgA is concentrated in the outer layer of colonic mucus along with gut bacteria. Pathogens. https://doi.org/10.3390/pathogens3020390

118. Bunker, J. J., \& Bendelac, A. (2018). IgA responses to microbiota. Immunity. https://doi.org/10.1016/j.immuni.2018.08.011

119. Hoces, D., Arnoldini, M., Diard, M., Loverdo, C., \& Slack, E. (2020). Growing, evolving and sticking in a flowing environment: understanding IgA interactions with bacteria in the gut. In Immunology. https://doi.org/10.1111/imm.13156

120. Macpherson, A. J. \& Smith, K. Mesenteric lymph nodes at the center of immune anatomy. J. Exp. Med. 203, 497-500 (2006).

121. Worbs, T. Oral tolerance originates in the intestinal immune system and relies on antigen carriage by dendritic cells. J. Exp. Med. 203, 519-527 (2006).

122. Mucida, D. et al. Oral tolerance in the absence of naturally occurring Tregs. J. Clin. Investig. 115, 1923-1933 (2005).

123. Scher, J. U., et al (2013). Expansion of intestinal Prevotella copri correlates with enhanced susceptibility to arthritis. ELife. https://doi.org/10.7554/elife.01202

124. Axelrad, J. E., Lichtiger, S., \& Yajnik, V. (2016). Inflammatory bowel disease and cancer: The role of inflammation, immunosuppression, and cancer treatment. In World J. Gastroenterol. https://doi.org/10.3748/wjg.v22.i20.4794

125. Som, A., et al (2019). Immune checkpoint inhibitor-induced colitis: a comprehensive review. In World J. Clin. Cases. https://doi.org/10.12998/wjcc.v7.i4.405

126. Chaput, N., et al (2017). Baseline gut microbiota predicts clinical response and colitis in metastatic melanoma patients treated with ipilimumab. Ann. Oncol. https://doi.org/10.1093/annonc/mdx108

127. Dubin, K., et al (2016). Intestinal microbiome analyses identify melanoma patients at risk for checkpoint-blockade-induced colitis. Nat. Commun. https:// doi.org/10.1038/ncomms10391

128. Vétizou, M., et al (2015). Anticancer immunotherapy by CTLA-4 blockade relies on the gut microbiota. Science. https://doi.org/10.1126/science.aad1329

129. Roberti, M. P., et al (2020). Chemotherapy-induced ileal crypt apoptosis and the ileal microbiome shape immunosurveillance and prognosis of proximal colon cancer. Nat. Med. https://doi.org/10.1038/s41591-020-0882-8

130. Al Nabhani, Z., et al (2019). A weaning reaction to microbiota is required for resistance to immunopathologies in the adult. Immunity. https://doi.org/ 10.1016/j.immuni.2019.02.014

131. Jabri, B., \& Sollid, L. M. (2009). Tissue-mediated control of immunopathology in coeliac disease. Nat. Rev. Immunol. 9. https://doi.org/10.1038/nri2670

132. Junker, Y., et al (2012). Wheat amylase trypsin inhibitors drive intestinal inflammation via activation of toll-like receptor 4. J. Exp. Med., 209. https://doi. org/10.1084/jem.20102660

133. Abadie, V., Sollid, L. M., Barreiro, L. B. \& Jabri, B. Integration of genetic and immunological insights into a model of celiac disease pathogenesis. Annu. Rev. Immunol. 29, 493-525 (2011).

134. Yu, W., Freeland, D. M. H., \& Nadeau, K. C. (2016). Food allergy: Immune mechanisms, diagnosis and immunotherapy. Nat. Rev. Immunol. (Vol. 16, Issue 12). https://doi.org/10.1038/nri.2016.111

135. Bublin, M., Eiwegger, T. \& Breiteneder, H. Do lipids influence the allergic sensitization process? J. Allergy Clin. Immunol. 134, 521-529 (2014). Issue 3.

136. Koppelman, S. J., Hefle, S. L., Taylor, S. L. \& de Jong, G. A. H. Digestion of peanut allergens Ara h 1, Ara h 2, Ara h 3, and Ara h 6: a comparative in vitro study and partial characterization of digestion-resistant peptide. Mol. Nutr. Food Res. 54, 1711-1721 (2010).

137. Balakireva, A. V., \& Zamyatnin, A. A. (2016). Properties of gluten intolerance: Gluten structure, evolution, pathogenicity and detoxification capabilities. In Nutrients (Vol. 8, Issue 10). https://doi.org/10.3390/nu8100644

138. Friedrich, M., Pohin, M., \& Powrie, F. (2019). Cytokine networks in the pathophysiology of inflammatory bowel disease. In Immunity. https://doi.org/10.1016/ j.immuni.2019.03.017

139. Sun, R., Hedl, M., \& Abraham, C. (2020). IL23 group> IL23R recycling and amplifies innate receptor-induced signalling and cytokines in human macrophages, and the IBD-protective IL23R R381Q variant modulates these outcomes. Gut, 69. https://doi.org/10.1136/gutjnl-2018-316830

140. Lee, J. S., et al (2015). Interleukin-23-independent IL-17 production regulates intestinal epithelial permeability. Immunity, 43. https://doi.org/10.1016/j. immuni.2015.09.003

141. Maxwell, J. R. et al (2015). Differential roles for interleukin-23 and interleukin17 in intestinal immunoregulation. Immunity, 43. https://doi.org/10.1016/j. immuni.2015.08.019 